\title{
IMAGES OF SEMISTABLE GALOIS REPRESENTATIONS
}

\author{
Kenneth A. Ribet \\ In memory of Olga Taussky-Todd
}

\section{Introduction.}

Let $p$ be an odd prime number. Suppose first that $E$ is a semistable elliptic curve over $\mathbf{Q}$. The action of $\operatorname{Gal}(\overline{\mathbf{Q}} / \mathbf{Q})$ on the group of $p$-division points of $E$ defines a representation

$$
\rho_{E, p}: \operatorname{Gal}(\overline{\mathbf{Q}} / \mathbf{Q}) \rightarrow \mathbf{G L}\left(2, \mathbf{F}_{p}\right) .
$$

Serre has shown that this representation is surjective whenever it is irreducible [26, Prop. 21], [29, §3.1]. Serre's arguments prove more generally the surjectivity of all continuous irreducible representations

$$
\rho: \operatorname{Gal}(\overline{\mathbf{Q}} / \mathbf{Q}) \rightarrow \mathbf{G L}\left(2, \mathbf{F}_{p}\right)
$$

which are semistable in the sense of $[15, \S 1]$. (Recall that $\rho$ is semistable if the determinant of $\rho$ is the $\bmod p$ cyclotomic character $\chi$, the Serre conductor of $\rho$ is square free, and the Serre weight of $\rho$ is either 2 or $p+1$. Here, the weight and conductor are the invariants defined in [28].)

In this article, we treat the situation where $\mathbf{F}_{p}$ is replaced by a finite field of characteristic $p$, or more generally by a finite product $\mathbf{F}=\prod F_{i}$ of finite fields of characteristic $p$. A continuous representation $\rho: \operatorname{Gal}(\overline{\mathbf{Q}} / \mathbf{Q}) \rightarrow$ $\mathbf{G L}(2, \mathbf{F})$ may then be viewed as a product of components $\rho_{i}: \operatorname{Gal}(\overline{\mathbf{Q}} / \mathbf{Q}) \rightarrow$ $\mathbf{G L}\left(2, F_{i}\right)$. We shall assume that each $\rho_{i}$ is irreducible and semistable. Since the determinant of each $\rho_{i}$ is then the $\bmod p$ cyclotomic character, the image of $\rho$ is contained in the group

$$
A:=\left\{M \in \mathbf{G L}(2, \mathbf{F}) \mid \operatorname{det} M \in \mathbf{F}_{p}^{*}\right\} .
$$

Making the supplementary hypothesis $p \geq 5$, we show below that the image of $\rho$ is $\mathbf{G L}(2, \mathbf{F})$-conjugate to

$$
\left\{M \in \mathbf{G L}\left(2, \mathbf{F}^{\prime}\right) \mid \operatorname{det} M \in \mathbf{F}_{p}^{*}\right\},
$$


where $\mathbf{F}^{\prime}$ is the subalgebra of $\mathbf{F}$ generated by the traces $\operatorname{tr}(\rho(\sigma))$ for $\sigma \in$ $\operatorname{Gal}(\overline{\mathbf{Q}} / \mathbf{Q})$. (See Theorem 3.2 and Corollary 3.3.) In particular, $\rho(\operatorname{Gal}(\overline{\mathbf{Q}} / \mathbf{Q}))=A$ if and only if $\mathbf{F}$ is generated as an $\mathbf{F}_{p}$-algebra by the traces.

Applying the lifting techniques of [25], we deduce an analogous result for certain $p$-adic representations of $\operatorname{Gal}(\overline{\mathbf{Q}} / \mathbf{Q})$.

Our results have evident relevance to the study of the Galois representations defined by semistable abelian varieties over $\mathbf{Q}$ which are products of abelian varieties with large fields of endomorphisms. (In [23], the author referred to these as abelian varieties of "GL $\mathbf{L}_{2}$ type.") While the computation of $\mathbf{F}^{\prime}$ seems to be difficult to perform in certain cases, it can be carried out for the $\bmod p$ representations coming from $J_{0}(N)$ if $N$ is a prime and $p$ is a prime number satisfying some mild conditions. In fact, our original goal was to find the image of the $p$-adic representation attached to $J_{0}(N)$, thus answering questions which were formulated by R. Coleman and B. Kaskel in connection with [10] and [2]. It is a pleasure to thank them for their continuing encouragement and interest in this work.

\section{Representations over a finite field.}

Let $\mathbf{F}$ be a finite field, and let $p$ be the characteristic of $\mathbf{F}$. We will assume that $p$ is odd; most of our results will require that $p$ be at least 5 . Suppose that

$$
\rho: \operatorname{Gal}(\overline{\mathbf{Q}} / \mathbf{Q}) \rightarrow \mathbf{G L}(2, \mathbf{F})
$$

is an irreducible representation whose determinant is the mod $p$ cyclotomic character $\chi: \operatorname{Gal}(\overline{\mathbf{Q}} / \mathbf{Q}) \rightarrow \mathbf{F}_{p}^{*} \hookrightarrow \mathbf{F}^{*}$. Let $k(\rho)$ and $N(\rho)$ be the weight and conductor of $\rho$ in the sense of Serre [28, $\S \S 1-2]$. As we recalled above, Oesterlé $[15, \S 1]$ has defined the notion of semistability: $\rho$ is semistable if the conductor $N(\rho)$ of $\rho$ is square free and Serre's weight $k(\rho)$ is either 2 or $p+1$.

The definition of the conductor shows that $N(\rho)$ is square free if and only if $\rho(\sigma)$ is unipotent whenever $\sigma$ belongs to an inertia subgroup of $\operatorname{Gal}(\overline{\mathbf{Q}} / \mathbf{Q})$ for a prime $\ell \neq p$. To illuminate the condition on $k(\rho)$, we let $I$ be an inertia subgroup of $\operatorname{Gal}(\overline{\mathbf{Q}} / \mathbf{Q})$ for the prime $p$. The semisimplification of $\rho_{\mid I}$ is described by a pair of characters $\varphi, \varphi^{\prime}: I \rightrightarrows \overline{\mathbf{F}}^{*}$, cf. [28, §2]. Since $\operatorname{det} \rho$ is the cyclotomic character $\chi$, we have in particular $\varphi \varphi^{\prime}=\chi$. If $k(\rho)$ is one of $2, p+1$, then $\left\{\varphi, \varphi^{\prime}\right\}$ is either $\{1, \chi\}$ or else the set of fundamental characters $\psi, \psi^{\prime}: I \rightarrow \overline{\mathbf{F}}^{*}$ of level $2([28, \S 2])$. It follows that the order of $\varphi^{\prime} \varphi^{-1}$ (a character which is defined only up to inversion) is either $p-1$ or $p+1$.

Lemma 2.1. Assume that $p>3$ and that $\rho$ is a semistable irreducible 
representation as above. Let $\epsilon: \operatorname{Gal}(\overline{\mathbf{Q}} / \mathbf{Q}) \rightarrow \mathbf{F}^{*}$ be a continuous character. If $\epsilon \otimes \rho$ is semistable, then $\epsilon$ is trivial.

Proof. By definition, the representation $\epsilon \otimes \rho$ sends each $\sigma \in \operatorname{Gal}(\overline{\mathbf{Q}} / \mathbf{Q})$ to the product $\epsilon(\sigma) \cdot \rho(\sigma) \in \mathbf{G} \mathbf{L}(2, \mathbf{F})$. If $\rho$ and $\epsilon \otimes \rho$ are both semistable, then $\epsilon$ is certainly unramified outside $p$, since both $\rho(\sigma)$ and $\epsilon(\sigma) \cdot \rho(\sigma)$ are unipotent whenever $\sigma$ belongs to an inertia group for a prime $\ell \neq p$. Moreover, $\epsilon^{2}$ is the trivial character, since the determinants of $\rho$ and $\epsilon \otimes \rho$ coincide. Hence $\epsilon$ is either the trivial character, as desired, or the quadratic character $\chi^{\frac{p-1}{2}}$. One checks easily, however, that no pair of characters drawn from the set $\left\{1, \chi, \psi, \psi^{\prime}\right\}$ have a quadratic ratio.

Remark. The lemma does not extend to cover the case $p=3$. To see this, consider the modular forms $f=\sum a_{n} q^{n}$ and $g=\sum b_{n} q^{n}$ which correspond to the two strong modular elliptic curves of conductor 89. As B. Gross explains in the last paragraphs of [8], these forms define irreducible mod 3 representations $\rho_{f}$ and $\rho_{g}$ of $\mathrm{Gal}(\overline{\mathbf{Q}} / \mathbf{Q})$ whose restrictions to a decomposition group for 3 are direct sums of two characters. Further, although $\rho_{f}$ and $\rho_{g}$ are semistable, the two representations are twists of each other by the mod 3 cyclotomic character. (In other words, $f$ and $g$ are "companions" of each other in Serre's language.)

To verify this latter fact, we can cite [8, Th. 13.10] along with Gross, or perform a numerical calculation with gp [1] to check that the mod 3 congruence $b_{p} \equiv\left(\frac{p}{3}\right) a_{p}$ holds for $3 \leq p \leq 200$. Using the results of J. Sturm [33], we can then conclude that it holds for all $p \geq 3$.

It perhaps is worth recalling at this juncture that an irreducible mod $p$ semistable representation $\rho$ is absolutely irreducible. This follows easily from the fact that $\rho(\operatorname{Gal}(\overline{\mathbf{Q}} / \mathbf{Q}))$ contains a matrix with distinct eigenvalues in $\mathbf{F}_{p}^{*}$. Such a matrix is obtained by taking $\rho(c)$, where $c$ is a complex conjugation - because det $\rho$ is the cyclotomic character, which is odd, the eigenvalues of $\rho(c)$ are +1 and -1 . These are distinct because $p$ is odd.

Proposition 2.2. If $\rho$ is semistable, then the image of $\rho$ has order divisible by $p$.

Proof. Let $G=\rho(\operatorname{Gal}(\overline{\mathbf{Q}} / \mathbf{Q}))$, and assume that the order of $G$ is prime to $p$. Since the semistability hypothesis implies that ramification subgroups of $G$ for primes $\ell \neq p$ are unipotent, these ramification groups are forced to be trivial. In other words, we have $N(\rho)=1$ and the representation $\rho$ is unramified outside $p$. As Serre remarks in a note on page 710 of [27, Vol. III], an analogue of the argument of Tate [34] shows that there are no irreducible representations $\rho$ with this property when $p=3$. 
Now assume that $p \geq 5$ and write $\bar{G}$ for the image of $G$ in $\operatorname{PGL}(2, \mathbf{F})$. Group theory shows that $\bar{G}$ is either cyclic or dihedral, or else one of the three exceptional groups $\mathbf{S}_{4}, \mathbf{A}_{4}, \mathbf{A}_{5}[26, \S 2.5]$. In fact, $\bar{G}$ cannot be cyclic, since the cyclicity of $\bar{G}$ would imply that $G$ is abelian, and hence that $\rho$ is not absolutely irreducible.

To rule out the other cases, one considers an inertia subgroup $\bar{I}$ of $\bar{G}$ for the prime $p$. We know that $\bar{I}$ is a cyclic group of order either $p+1$ or $p-1$. Indeed, $\bar{I}$ may be viewed as the image of $I \subset \operatorname{Gal}(\overline{\mathbf{Q}} / \mathbf{Q})$ under the character $\varphi / \varphi^{\prime}$ which we introduced above. This character has order either $p-1$ or $p+1$; its image is cyclic because it is a finite subgroup of $\overline{\mathbf{F}}^{*}$.

Assume now that $\bar{G}$ is dihedral, and let $Z$ be the center of $\bar{G}$. It is evident that $\bar{I}$ is contained in $Z$, since $\bar{I}$ is a cyclic subgroup of a dihedral group and the order of $\bar{I}$ is greater than 2. Accordingly, the quadratic extension of $\mathbf{Q}$ corresponding to $Z$ is everywhere unramified - this contradiction excludes the dihedral case and shows that $\bar{G}$ must be one of the three exceptional groups.

However, as observed in the proof of [26, Prop. 21], the fact that $\bar{I}$ has an element of order $p \pm 1$ rules out the groups $\mathbf{S}_{4}, \mathbf{A}_{4}, \mathbf{A}_{5}$ in case $p \geq 7$. Thus we are left only with the possibility that $p=5$, in which case $\bar{G}$ is either $\mathbf{S}_{4}$ or $\mathbf{A}_{4}$, since its order is prime to 5 by assumption. The group $\bar{I}$ is then cyclic of order 4 , since $\mathbf{S}_{4}$ has no element of order 6 . Also, we have $\bar{G} \approx \mathbf{S}_{4}$, since $\mathbf{A}_{4}$ has no element of order 4 . Consider the quotient $\mathbf{S}_{3}$ of $\mathbf{S}_{4}$. This quotient allows us to produce an $\mathbf{S}_{3}$-extension of $\mathbf{Q}$ which is ramified only at 5 and such that the inertia groups for 5 in the extension have order 2. However, there certainly is no such extension, since the class number of $\mathbf{Q}(\sqrt{5})$ is 1 .

Corollary 2.3 (cf. [29, Prop. 1]). Let $\rho$ be as in Proposition 2.2, and suppose that $p>2$. Then the image of $\rho$ contains a subgroup isomorphic to $\mathbf{S L}\left(2, \mathbf{F}_{p}\right)$. In particular, if $\mathbf{F}=\mathbf{F}_{p}$, then $\rho$ is surjective.

Proof. The second statement is a consequence of the first, since the cyclotomic character $\chi: \operatorname{Gal}(\overline{\mathbf{Q}} / \mathbf{Q}) \rightarrow \mathbf{F}_{p}^{*}$ is surjective. To prove the first statement, we proceed as in $[26, \S 2.4]$. Namely, let $g \in G$ be an element of order $p$, and let $v$ be a non-zero vector in $\mathbf{F} \oplus \mathbf{F}$ which is fixed by $g$. Since $\rho$ is irreducible, $G$ cannot fix the line generated by $v$; therefore, there is an $r \in G$ such that $v$ and $r v$ form a basis of $\mathbf{F} \oplus \mathbf{F}$. With respect to this basis, $g$ has the form $\left(\begin{array}{ll}1 & a \\ 0 & 1\end{array}\right)$, while $\operatorname{rgr}^{-1}$ has the form $\left(\begin{array}{ll}1 & 0 \\ b & 1\end{array}\right)$. Scaling one of the vectors $v, r v$, we may assume that $a=1$. Hence, in an appropriate basis, $G$ contains the elements $\left(\begin{array}{ll}1 & 1 \\ 0 & 1\end{array}\right)$ and $\left(\begin{array}{ll}1 & 0 \\ b & 1\end{array}\right)$. A well known theorem 
of L. E. Dickson (cf. [7, Th. 2.8.4]) states that the group generated by these elements has a subgroup isomorphic to $\mathbf{S L}\left(2, \mathbf{F}_{p}\right)$. In fact, a more precise statement is true for $p \neq 3:\left(\begin{array}{ll}1 & 1 \\ 0 & 1\end{array}\right)$ and $\left(\begin{array}{ll}1 & 0 \\ b & 1\end{array}\right)$ generate the group $\mathbf{S L}\left(2, \mathbf{F}^{\prime}\right)$, where $\mathbf{F}^{\prime}=\mathbf{F}_{p}(b)$.

Our aim now is to complement the Corollary by determining the exact image of $\rho$ in a situation generalizing that where $\mathbf{F}=\mathbf{F}_{p}$. The situation which we have in mind is that where $\mathbf{F}$ is a minimal field of definition for $\rho$ in the sense that it is generated over $\mathbf{F}_{p}$ by the numbers $\operatorname{tr}(\rho(\sigma))$ for $\sigma \in \operatorname{Gal}(\overline{\mathbf{Q}} / \mathbf{Q})$. We first show in this case that $\mathbf{F}$ is also a minimal field of definition for $\rho$ as a projective representation, at least when $p \geq 5$.

For the following lemma, let $\overline{\mathbf{F}}$ be an algebraic closure of $\mathbf{F}$ and consider an arbitrary subfield $K$ of $\overline{\mathbf{F}}$. We view $G$ and $\bar{G}$ inside $\mathbf{G L}(2, \overline{\mathbf{F}})$ and $\mathbf{P G L}(2, \overline{\mathbf{F}})$, respectively.

Lemma 2.4. Suppose that $\rho$ semistable and that $p \geq 5$. Then $\bar{G}$ lies in $\mathbf{P G L}(2, K)$ if and only if $G$ lies in $\mathbf{G L}(2, K)$.

Proof. If $G$ lies in $\mathbf{G L}(2, K)$, then it is evident that $\bar{G}$ is contained in $\mathbf{P G L}(2, K)$. Conversely, suppose $\bar{G} \subseteq \mathbf{P G L}(2, K)$. Then certainly $G \subseteq$ $\overline{\mathbf{F}}^{*} \cdot \mathbf{G L}(2, K)$. Let $\alpha: \operatorname{Gal}(\overline{\mathbf{Q}} / \mathbf{Q}) \rightarrow \overline{\mathbf{F}}^{*} / K^{*}$ be the composite homomorphism

$$
\operatorname{Gal}(\overline{\mathbf{Q}} / \mathbf{Q}) \stackrel{\rho}{\rightarrow} G \hookrightarrow \overline{\mathbf{F}}^{*} \mathbf{G L}(2, K) \rightarrow\left(\overline{\mathbf{F}}^{*} \mathbf{G L}(2, K)\right) / \mathbf{G L}(2, K)=\overline{\mathbf{F}}^{*} / K^{*}
$$

For $\sigma \in \operatorname{Gal}(\overline{\mathbf{Q}} / \mathbf{Q})$, we have $\alpha(\sigma)=1$ whenever the trace of $\rho(\sigma)$ is a nonzero element of $K$. Indeed, write $\rho(\sigma)=t \cdot M$, with $M \in \mathbf{G L}(2, K)$ and $t \in \overline{\mathbf{F}}^{*}$. Then $\operatorname{tr}(\rho(\sigma))=t \cdot \operatorname{tr}(M)$ and we have $\operatorname{tr} M \in K$. If $\operatorname{tr}(\rho(\sigma))$ belongs to $K^{*}$, then $t$ lies in $K$, so that $\rho(\sigma)$ is an element of $\mathbf{G L}(2, K)$. In particular, $\alpha(\sigma)=1$ whenever the trace of $\rho(\sigma)$ is a non-zero element of $\mathbf{F}_{p}$.

Let $M$ now be the finite abelian extension of $\mathbf{Q}$ which is cut out by $\alpha$. We seek to show that $\alpha$ is identically 1, i.e., that $M=\mathbf{Q}$. We first prove that $\alpha$ is unramified outside $p$ by using the remark about traces. If $\sigma$ belongs to an inertia subgroup of $\operatorname{Gal}(\overline{\mathbf{Q}} / \mathbf{Q})$ for a prime $\ell \neq p$, then $\rho(\sigma)$ is unipotent, so that its trace is 2 . Since $p$ is odd, 2 is a non-zero element of $\mathbf{F}_{p}$, and we may conclude that $\alpha(\sigma)=1$.

Thus $M$ is a finite abelian extension of $\mathbf{Q}$ which is unramified outside $p$. Moreover, $[M: \mathbf{Q}]$ is prime to $p$, since $\overline{\mathbf{F}}^{*}$ has no elements of order $p$. Hence one has $M \subseteq \mathbf{Q}\left(\mu_{p}\right)$; equivalently, $\alpha$ factors through the $\bmod p$ cyclotomic character $\chi$.

Now let $I$ be an inertia group for $p$ in $\operatorname{Gal}(\overline{\mathbf{Q}} / \mathbf{Q})$. It suffices to show that there is an element $\sigma$ of $I$ for which $\alpha(\sigma)=1$ and for which $\chi(\sigma)$ is a 
generator of the cyclic group $\mathbf{F}_{p}^{*}$. The semisimplification of $\rho_{\mid I}$ is described by a pair of characters $\varphi, \varphi^{\prime}: I \rightrightarrows \overline{\mathbf{F}}^{*}$, cf. $[28, \S 2]$. As we mentioned above, one has either $\left\{\varphi, \varphi^{\prime}\right\}=\{1, \chi\}$ or $\left\{\varphi, \varphi^{\prime}\right\}=\left\{\psi, \psi^{p}\right\}$, where $\psi$ and $\psi^{p}$ are the fundamental characters of level 2. Suppose first that we are in the former case, and let $\sigma \in I$ be such that $t=\chi(\sigma)$ is a generator of $\mathbf{F}_{p}^{*}$. Then $\operatorname{tr}(\rho(\sigma))=1+t$ is non-zero since $p \neq 3$. Thus $\alpha(\sigma)=1$, as required. In the latter case, choose $\sigma \in I$ so that $t=\psi(\sigma)$ generates $\psi(I) \approx \mathbf{F}_{p^{2}}$. Then $\chi(\sigma)=t^{p+1}$ is a generator of $\mathbf{F}_{p}^{*}$; note that $\chi=\psi \psi^{\prime}=\psi^{p+1}$. On the other hand, $\operatorname{tr}(\rho(\sigma))=t+t^{p}$. The number $t^{p-1}$ cannot be -1 , since it has order $p+1$. Since $\operatorname{tr}(\rho(\sigma))$ is non-zero, we may conclude $\alpha(\sigma)=1$.

Theorem 2.5. Assume that $\rho$ is semistable, that $p \geq 5$ and that $\mathbf{F}$ is generated over $\mathbf{F}_{p}$ by the set $\{\operatorname{tr}(\rho(\sigma)) \mid \sigma \in \operatorname{Gal}(\overline{\mathbf{Q}} / \mathbf{Q})\}$. Then

$$
\rho(\operatorname{Gal}(\overline{\mathbf{Q}} / \mathbf{Q}))=\left\{M \in \mathbf{G L}(2, \mathbf{F}) \mid \operatorname{det} M \in \mathbf{F}_{p}^{*}\right\} .
$$

Proof. It is clear that $G=\rho(\operatorname{Gal}(\overline{\mathbf{Q}} / \mathbf{Q}))$ is contained in the indicated matrix group because $\operatorname{det} \rho$ is $\mathbf{F}_{p}^{*}$-valued. Since the image of $\operatorname{det} \rho$ is precisely $\mathbf{F}_{p}^{*}$, the theorem amounts to the statement that $G$ contains $\mathbf{S L}(2, \mathbf{F})$. An equivalent assertion is that the commutator subgroup of $G$ contains a subgroup isomorphic to $\mathbf{S L}(2, \mathbf{F})$.

Let $k$ be a finite extension of $\mathbf{F}_{p}$ which contains $\mathbf{F}$ and which has even degree over $\mathbf{F}_{p}$. Since $\operatorname{det}(G) \subseteq \mathbf{F}_{p}^{*}$, the subgroup $\bar{G}$ of $\mathbf{P G L}(2, k)$ lies in $\mathbf{P S L}(2, k)$. Dickson [4, Ch. XII] has enumerated all subgroups of $\operatorname{PSL}(2, k)$; his list is summarized in $[4, \S 260]$.

To situate $\bar{G}$ within Dickson's list, we recall that the order of $\bar{G}$ is divisible by $p$ by Proposition 2.2 , and that the identity representation $G \rightarrow \mathbf{G L}(2, \overline{\mathbf{F}})$ is irreducible. (The representation $\rho$ is irreducible over $\mathbf{F}$ by hypothesis. It is then absolutely irreducible, as was noted above.) It follows that $\bar{G}$ is one of the groups enumerated in $[4, \S 251-\S 253]$. Since we have assumed $p \geq 5$, the final conclusion is easy to state: After replacing $\bar{G}$ by a conjugate of $\bar{G}$ inside $\mathbf{P G L}(2, k)$, we have either $\bar{G}=\mathbf{P S L}(2, K)$ or $\bar{G}=\mathbf{P G L}(2, K)$, for some subfield $K$ of $k$.

Thus, in either case one has $\bar{G} \subseteq \mathbf{P G L}(2, K)$. From the Lemma, $G \subseteq$ $\mathbf{G L}(2, K)$. In particular, $\operatorname{tr}(\rho(\sigma)) \in K$ for all $\sigma \in \operatorname{Gal}(\overline{\mathbf{Q}} / \mathbf{Q})$. Since these numbers generate $\mathbf{F}$ over $\mathbf{F}_{p}, \mathbf{F} \subseteq K$. On the other hand, $\mathbf{S L}(2, K) \subseteq$ $k^{*} \cdot G$ because $\bar{G}$ contains PSL $(2, K)$. On taking commutators, we obtain $\mathbf{S L}(2, K) \subseteq[G, G]$, and therefore $\mathbf{S L}(2, F) \subseteq[G, G]$. As indicated above, this proves the theorem.

In the spirit of $[26$, Th. 4], let us choose an inertia group $I$ for $p$ in $\operatorname{Gal}(\overline{\mathbf{Q}} / \mathbf{Q})$ and define $X$ to be the smallest closed normal subgroup of $\mathrm{Gal}(\overline{\mathbf{Q}} / \mathbf{Q})$ which contains $I$. 
Theorem 2.6. In the situation of Theorem 2.5

$$
\rho(X)=\left\{M \in \mathbf{G L}(2, \mathbf{F}) \mid \operatorname{det} M \in \mathbf{F}_{p}^{*}\right\} .
$$

Proof. By Theorem 2.5, the group $G=\rho(\operatorname{Gal}(\overline{\mathbf{Q}} / \mathbf{Q}))$ is the group of matrices in $\mathbf{G L}(2, \mathbf{F})$ with determinants in $\mathbf{F}_{p}^{*}$. Let $H=\rho(X)$; thus $H$ is a normal subgroup of $G$. Let $\bar{H}$ and $\bar{G}$ be the images of $H$ and $G$ in PGL $(2, \mathbf{F})$. The group $\bar{G}$ is either $\mathbf{P S L}(2, \mathbf{F})$ or $\mathbf{P G L}(2, \mathbf{F})$, according as the degree of $\mathbf{F}$ over $\mathbf{F}_{p}$ is even or odd. The discussion above shows that the order of $H$ is at least $p-1 \geq 4$. Therefore, the intersection $H \cap \mathbf{P S L}(2, \mathbf{F})$ has order at least 2. Since $\mathbf{P S L}(2, \mathbf{F})$ is normal in $\bar{G}, H \cap \mathbf{P S L}(2, \mathbf{F})$ is a non-trivial normal subgroup of $\mathbf{P S L}(2, \mathbf{F})$. Accordingly, it is all of $\mathbf{P S L}(2, \mathbf{F})$; in other words, $\bar{H}$ contains PSL $(2, \mathbf{F})$. On taking commutators as above, we see that $H$ contains $\mathbf{S L}(2, \mathbf{F})$. Because the $\bmod p$ cyclotomic character maps $I$ onto $\mathbf{F}_{p}^{*}$, it follows now that $H=G$.

Remark 1. In the context of Theorem 2.6, one may consider the more general situation where $\mathbf{F}$ is not necessarily generated by the traces $\operatorname{tr}(\rho(\sigma))$ for $\sigma \in \operatorname{Gal}(\overline{\mathbf{Q}} / \mathbf{Q})$. Let $\mathbf{F}^{\prime}$ be the subfield of $\mathbf{F}$ which is generated by these traces. Because complex conjugation acts in $\rho$ as a matrix with distinct rational eigenvalues, a well known theorem of I. Schur [24, IX a] (cf. [35, Lemme I.1]) implies that $\rho$ can be conjugated into a representation with values in $\mathbf{G L}\left(2, \mathbf{F}^{\prime}\right)$. The theorem applies to this latter representation, and shows that its image is the group of matrices in $\mathbf{G L}\left(2, \mathbf{F}^{\prime}\right)$ whose determinants lie in the multiplicative group of the prime field $\mathbf{F}_{p}$.

To prove the well known statement that there is a model for $\rho$ over $\mathbf{F}^{\prime}$, one may proceed alternatively by direct computation, along the lines suggested by Wiles [36, p. 483].

Remark 2. The referee has asked whether Theorem 2.5 extends to the case $p=3$. The answer is negative; in fact, a counterexample is furnished by the abelian surface $J_{0}(23)$. Recall that $J_{0}(23)$ has "real multiplication" by the Hecke ring $\mathbf{T}$ associated with the space of weight-two cusp forms on $\Gamma_{0}(23)$. The algebra $\mathbf{T} \otimes \mathbf{Q}$ is the real quadratic field $\mathbf{Q}(\sqrt{5})$, and $\mathbf{T}$ is the ring of integers of $\mathbf{T} \otimes \mathbf{Q}$. The group $V$ of 3 -division points on $J_{0}(23)$ is a twodimensional vector space over the field $\mathbf{F}:=\mathbf{T} / 3 \mathbf{T}$, which has nine elements. The action of $\operatorname{Gal}(\overline{\mathbf{Q}} / \mathbf{Q})$ on $V$ is given by a homomorphism $\rho: \operatorname{Gal}(\overline{\mathbf{Q}} / \mathbf{Q}) \rightarrow$ Aut $_{\mathbf{F}} V \approx \mathbf{G} \mathbf{L}(2, \mathbf{F})$. As we shall see in $\S 5$, this representation is semistable; it is irreducible because 3 is prime to $23-1$. Moreover, the traces $\operatorname{tr}(\rho(\sigma))$ generate $\mathbf{F}$; indeed, the trace of a Frobenius element Frob $_{2}$ for the prime 2 satisfies $x^{2}+x-1=0$. In view of Theorem 2.5 , it is very tempting to guess that the image of $\rho$ contains $\mathbf{S L}(2, \mathbf{F})$. Equivalently, consider the composite 
of $\rho$ and the natural homomorphism $\mathbf{G L}(2, \mathbf{F}) \rightarrow \mathbf{P G L}(2, \mathbf{F})$; let $\bar{G}$ be the image of this composite, so that $\bar{G}$ is a subgroup of $\mathbf{P S L}(2, \mathbf{F})$. The guess that the image of $\rho$ contains $\mathbf{S L}(2, \mathbf{F})$ means that $\bar{G}=\mathbf{P S L}(2, \mathbf{F})$.

What information do we have about $\bar{G}$ ? The group $\bar{G}$ is "irreducible" (i.e., acts transitively on $\left.\mathbf{P}^{1}(\mathbf{F})\right)$ since $\rho$ is irreducible. Also, the order of $\bar{G}$ is divisible by 3 , as one sees by considering an inertia subgroup of $G$ for the prime 23. Finally, the order of $\bar{G}$ is divisible by 5 because of the information concerning $\operatorname{tr}\left(\rho\left(\right.\right.$ Frob $\left.\left._{2}\right)\right)$, which implies that $\rho\left(\right.$ Frob $\left._{2}\right)$ has order 5 . The results of Dickson used above thus permit only two possibilities for $\bar{G}$ : either $\bar{G}$ is all of $\mathbf{P S L}(2, \mathbf{F})$, or else the alternating group $\mathbf{A}_{5}$.

Rather to the author's surprise, calculations based on [14, Table B] suggested strongly that $\bar{G}$ is in fact the smaller of these two groups. The author's suspicion that this was the case deepened when he learned that there is an $\mathbf{A}_{5}$-extension of $\mathbf{Q}$ which is ramified only at 3 and 23: the second line of [6, Table 1] shows that the splitting field of the polynomial $x^{5}+3 x^{3}+6 x^{2}+9$ is such an extension. Subsequently, Jean-François Mestre carried out computations which confirm that this latter $\mathbf{A}_{5}$-extension is indeed the extension of $\mathbf{Q}$ which is cut out by the projective representation deduced from $\rho$. In particular, one has $\bar{G} \approx \mathbf{A}_{5}$.

\section{Products.}

We next consider finite products of representations as above, keeping fixed the prime number $p$. Thus let $F_{1}, \ldots, F_{t}(t \geq 1)$ be finite fields of characteristic $p$, where $p$ is a prime which is different from 2 and 3 . Let $\mathbf{F}$ be the finite étale $\mathbf{F}_{p}$-algebra $F_{1} \times \cdots \times F_{t}$. Suppose that $\rho: \operatorname{Gal}(\overline{\mathbf{Q}} / \mathbf{Q}) \rightarrow \mathbf{G L}(2, \mathbf{F})$ is a continuous homomorphism, so that $\rho$ is a product of representations $\rho_{i}$ $(i=1, \ldots, t)$ as above. We will assume that each $\rho_{i}$ is semistable and irreducible, and also that $\operatorname{det} \rho_{i}=\chi$ for $i=1, \ldots, t$. With the evident convention, the latter hypothesis may be summarized by the formula $\operatorname{det} \rho=\chi$.

For each $\sigma \in \operatorname{Gal}(\overline{\mathbf{Q}} / \mathbf{Q})$, we obtain an element $\operatorname{tr}(\rho(\sigma))$ of $\mathbf{F}$ by considering the trace of the matrix $\rho(\sigma)$. Motivated by the remark above, we let $\mathbf{F}^{\prime}$ be the subalgebra of $\mathbf{F}$ generated by the $\operatorname{tr}(\rho(\sigma))$.

Lemma 3.1. The representation $\rho$ has a model over $\mathbf{F}^{\prime}$.

Proof. As we have seen, each component $\rho_{i}$ has a model over the subfield of $F_{i}$ generated by the traces $\operatorname{tr}\left(\rho_{i}(\sigma)\right)$. After replacing $\rho_{i}$ by such a model (and $F_{i}$ by the trace field in question), we arrive at a situation in which $\mathbf{F}^{\prime}$ maps surjectively onto each factor $F_{i}$.

As one knows, $\mathbf{F}^{\prime}$ is then isomorphic to a partial product of the factors $F_{i}$. To see this explicitly, we let $\pi: \mathbf{F} \rightarrow F_{i}$ be the projections $\left(x_{1}, \ldots, x_{t}\right) \mapsto x_{i}$ 
and consider the following relation on the set $\{1, \ldots, t\}: i \sim j$ if and only if the map $\pi_{i} \times \pi_{j}: \mathbf{F}^{\prime} \rightarrow F_{i} \times F_{j}$ is not surjective. It is easy to see that this relation is an equivalence relation and that $i \sim j$ if and only if there is an isomorphism $\sigma: F_{i} \stackrel{\sim}{\rightarrow} F_{j}$ so that $\pi_{j}=\sigma \circ \pi_{i}$ on $\mathbf{F}^{\prime}$. If there is such an isomorphism, it is unique; we denote it $\sigma_{j i}$. One shows that

$$
\mathbf{F}^{\prime}=\left\{\left(x_{1}, \ldots, x_{t}\right) \in \mathbf{F} \mid x_{j}=\sigma_{j i}\left(x_{i}\right) \text { for all pairs }(i, j) \text { such that } i \sim j\right\} .
$$

In particular, $\mathbf{F}^{\prime}$ is isomorphic to the product $\prod_{i \in I} F_{i}$, where $I$ is a set of representatives for the equivalence $\sim$.

By the Brauer-Nesbitt theorem, $\rho_{j}$ and ${ }^{\sigma_{j i}} \rho_{i}$ are isomorphic whenever $i$ and $j$ are equivalent. (The two representations have the same trace and determinant.) Replace $\rho_{j}$ by ${ }^{\sigma_{j i}} \rho_{i}$ for all equivalent pairs $(i, j)$ with $i \in I$. Then the representation $\rho$, a priori with values in $\mathbf{G L}(2, \mathbf{F})$, takes values in $\mathbf{G L}\left(2, \mathbf{F}^{\prime}\right)$.

Theorem 3.2. One has

$$
\rho(\operatorname{Gal}(\overline{\mathbf{Q}} / \mathbf{Q}))=\left\{M \in \mathbf{G L}(2, \mathbf{F}) \mid \operatorname{det} M \in \mathbf{F}_{p}^{*}\right\}
$$

if and only if $\mathbf{F}^{\prime}=\mathbf{F}$.

Proof. The necessity is clear, since the trace function $\mathbf{S L}(2, \mathbf{F}) \rightarrow \mathbf{F}$ is surjective. For the sufficiency, as in the proof of Theorem 2.5, one must show that $\rho(\operatorname{Gal}(\overline{\mathbf{Q}} / \mathbf{Q}))$ contains $\mathbf{S L}(2, \mathbf{F})$. Let $H=\rho(\operatorname{Gal}(\overline{\mathbf{Q}} / \mathbf{Q})) \cap \mathbf{S L}(2, \mathbf{F})$, so that $H$ is a subgroup of the product $\mathbf{S L}\left(2, F_{1}\right) \times \cdots \times \mathbf{S L}\left(2, F_{t}\right)$. By Theorem 2.5, $H$ projects onto each factor $\mathbf{S L}\left(2, F_{i}\right)$. Because each group $\mathbf{S L}\left(2, F_{i}\right)$ is its own commutator subgroup, the "two principle" [19,3.3] implies that $H$ is the full product of the $\mathbf{S L}\left(2, F_{i}\right)$ if and only if $H$ maps onto each product $\mathbf{S L}\left(2, F_{i}\right) \times \mathbf{S L}\left(2, F_{j}\right)$ for $i \neq j$.

Assume, then, that we have $i \neq j$, and suppose that the image of the product $\rho_{i} \times \rho_{j}$ does not contain $\mathbf{S L}\left(2, F_{i}\right) \times \mathbf{S L}\left(2, F_{j}\right)$. By exploiting results of Dieudonné and Hua, one may construct: (i) An isomorphism $\omega: F_{i} \stackrel{\sim}{\rightarrow}$ $F_{j}$, and (ii) a continuous homomorphism $\epsilon: \operatorname{Gal}(\overline{\mathbf{Q}} / \mathbf{Q}) \rightarrow F_{j}^{*}$ such that ${ }^{\omega} \rho_{i}$ and $\rho_{j} \otimes \epsilon$ are isomorphic representations of $\operatorname{Gal}(\overline{\mathbf{Q}} / \mathbf{Q})$ over $F_{j}$. This statement was proved during the course of the proof of [19, Th. 3.8] the theorem itself states merely that ${ }^{\omega} \rho_{i}$ and $\rho_{j} \otimes \epsilon$ have equal traces and determinants.

By Lemma 3.1, however, we have $\epsilon=1$; thus ${ }^{\omega} \rho_{i} \approx \rho_{j}$. Accordingly, we have $\omega\left(\operatorname{tr}\left(\rho_{i}(\sigma)\right)\right)=\operatorname{tr}\left(\rho_{j}(\sigma)\right)$ for all $\sigma \in \operatorname{Gal}(\overline{\mathbf{Q}} / \mathbf{Q})$. This means that the image of $\operatorname{tr}(\rho(\sigma))$ in $F_{i} \times F_{j}$ lies in the subalgebra $\left\{(x, \omega x) \mid x \in F_{i}\right\}$ of $F_{i} \times F_{j}$. Thus the quantities $\operatorname{tr}(\rho(\sigma))$ fail to generate $F_{i} \times F_{j}$ over $\mathbf{F}_{p}$. This contradicts the hypothesis that they generate the full product $\mathbf{F}$ over $\mathbf{F}_{p}$. 
Corollary 3.3. Assume that $\rho$ is as in Theorem 3.2. Then the image of $\rho$ is conjugate in $\mathbf{G L}(2, \mathbf{F})$ to the group

$$
\left\{M \in \mathbf{G L}\left(2, \mathbf{F}^{\prime}\right) \mid \operatorname{det} M \in \mathbf{F}_{p}^{*}\right\}
$$

Proof. By Lemma 3.1, there is a model for $\rho$ over $\mathbf{F}^{\prime}$ : after conjugating by a matrix in $\mathbf{G L}(2, \mathbf{F})$, we make $\rho$ take values in $\mathbf{G L}\left(2, \mathbf{F}^{\prime}\right)$. Applying the Theorem to this model, we arrive at the desired conclusion.

We continue the discussion begun with Theorem 2.6, letting $X$ be the subgroup of $\operatorname{Gal}(\overline{\mathbf{Q}} / \mathbf{Q})$ which appears in the statement of that result.

Theorem 3.4. In the situation of Theorem 3.2, suppose that $\mathbf{F}=\mathbf{F}^{\prime}$. Then $\rho(X)=\left\{M \in \mathbf{G L}(2, \mathbf{F}) \mid \operatorname{det} M \in \mathbf{F}_{p}^{*}\right\}$.

Proof. The group $\rho(X)$ is a normal subgroup of $G=\rho(\operatorname{Gal}(\overline{\mathbf{Q}} / \mathbf{Q}))$. By Theorem 3.2, $G=\left\{M \in \mathbf{G L}(2, \mathbf{F}) \mid \operatorname{det} M \in \mathbf{F}_{p}^{*}\right\}$. The intersection $\rho(X) \cap$ $\mathbf{S L}(2, \mathbf{F})$ is then normal in $\mathbf{S L}(2, \mathbf{F})$; it maps onto each factor $\mathbf{S L}\left(2, F_{i}\right)$ by Theorem 2.6. On taking commutators with elements of $\mathbf{S L}(2, \mathbf{F})$ of the form $(1, \ldots, 1, \alpha, 1, \ldots, 1)$, we see that $\rho(X) \cap \mathbf{S L}(2, \mathbf{F})$ contains $\mathbf{S L}\left(2, F_{i}\right)$ (viewed as a subgroup of $\mathbf{S L}(2, \mathbf{F})$ ) for each $i$. It follows that $\rho(X)$ contains $\mathbf{S L}(2, \mathbf{F})$. We then obtain $\rho(X)=G$, since the mod $p$ cyclotomic character is totally ramified at $p$.

\section{Lifts.}

Again suppose that $p$ is a prime $\geq 5$ and let $\mathcal{O}_{1}, \ldots, \mathcal{O}_{t}$ be integer rings of finite-degree unramified extensions of $\mathbf{Q}_{p}$. For each $i$, let $\tilde{\rho}_{i}: \operatorname{Gal}(\overline{\mathbf{Q}} / \mathbf{Q}) \rightarrow$ $\mathbf{G L}\left(2, \mathcal{O}_{i}\right)$ be a continuous representation whose determinant is the $p$-adic cyclotomic character $\tilde{\chi}: \operatorname{Gal}(\overline{\mathbf{Q}} / \mathbf{Q}) \rightarrow \mathbf{Z}_{p}^{*}$. Let $\tilde{\rho}$ be the product of the $\tilde{\rho}_{i}$, so that $\tilde{\rho}$ is a $p$-adic representation $\operatorname{Gal}(\overline{\mathbf{Q}} / \mathbf{Q}) \rightarrow \mathbf{G L}(2, \mathcal{O})$, where $\mathcal{O}:=\prod \mathcal{O}_{i}$. The diagonal embedding $\mathbf{Z}_{p} \hookrightarrow \mathcal{O}$ induces an inclusion $\mathbf{Z}_{p}^{*} \hookrightarrow \mathcal{O}^{*}$. We clearly have $\tilde{\rho}(\operatorname{Gal}(\overline{\mathbf{Q}} / \mathbf{Q})) \subseteq \mathcal{A}$, where

$$
\mathcal{A}=\left\{M \in \mathbf{G L}(2, \mathcal{O}) \mid \operatorname{det} M \in \mathbf{Z}_{p}^{*}\right\} .
$$

Let $\mathbf{F}=\mathcal{O} / p \mathcal{O}$, and let $\rho: \operatorname{Gal}(\overline{\mathbf{Q}} / \mathbf{Q}) \rightarrow \mathbf{G L}(2, \mathbf{F})$ be the reduction of $\tilde{\rho}$ $\bmod p$.

In the following statement, $X$ is again the closed normal subgroup of $\operatorname{Gal}(\overline{\mathbf{Q}} / \mathbf{Q})$ which is generated by the inertia groups for $p$ in $\operatorname{Gal}(\overline{\mathbf{Q}} / \mathbf{Q})$. 
Theorem 4.1. Suppose that the mod p reduction of each $\tilde{\rho}_{i}$ is semistable and irreducible. Assume that $\mathbf{F}$ is generated as an $\mathbf{F}_{p}$-algebra by the traces $\operatorname{tr} \rho(g)$ with $g \in \operatorname{Gal}(\overline{\mathbf{Q}} / \mathbf{Q})$. Then $\tilde{\rho}(X)=\tilde{\rho}(\operatorname{Gal}(\overline{\mathbf{Q}} / \mathbf{Q}))=\mathcal{A}$.

Remark. Using Nakayama's Lemma, one may reformulate the trace hypothesis as the apparently stronger assertion that $\mathcal{O}$ is generated as a $\mathbf{Z}_{p^{-}}$ algebra by the traces $\operatorname{tr} \tilde{\rho}(g)$ with $g \in \operatorname{Gal}(\overline{\mathbf{Q}} / \mathbf{Q})$.

Proof. The group $\tilde{\rho}(\operatorname{Gal}(\overline{\mathbf{Q}} / \mathbf{Q}))$ and its subgroup $\tilde{\rho}(X)$ are closed subgroups of $\mathcal{A}$ whose determinants are equal to all of $\mathbf{Z}_{p}^{*}$. It suffices to show that $\tilde{\rho}(X)$ contains $\mathbf{S L}(2, \mathcal{O})$. We recall the following fact (see [25, p. IV-23] and [19, Th. 2.1]):

Proposition 4.2. Let $\mathcal{G}$ be a closed subgroup of $\mathbf{G L}(2, \mathcal{O})$ and let $\overline{\mathcal{G}}$ be the image of $\mathcal{G}$ in $\mathbf{G L}(2, \mathcal{O} / p \mathcal{O})$. If $\overline{\mathcal{G}}$ contains $\mathbf{S L}(2, \mathcal{O} / p \mathcal{O})$, then $\mathcal{G}$ contains $\mathbf{S L}(2, \mathcal{O})$.

Proof. On taking $\mathcal{G}=\tilde{\rho}(X)$, we now obtain the required inclusion $\tilde{\rho}(X) \supseteq$ $\mathbf{S L}(2, \mathcal{O})$ from Theorem 3.4 .

\section{Semistable abelian varieties of $\mathrm{GL}_{2}$-type over $\mathrm{Q}$.}

Let $A$ be an abelian variety over $\mathbf{Q}$ for which $\mathbf{Q} \otimes \operatorname{End}_{\mathbf{Q}} A$ is a number field of degree equal to the dimension of $A$. Suppose that the $\operatorname{ring} R=\operatorname{End}_{\mathbf{Q}} A$ is the full ring of integers in the field $\mathbf{Q} \otimes \operatorname{End}_{\mathbf{Q}} A$. (After replacement of $A$ by an isogenous abelian variety, this hypothesis is always verified.) Let $\mathfrak{m}$ be a maximal ideal of $R$ and let $A[\mathfrak{m}]$ be the kernel of $\mathfrak{m}$ on $A$, i.e., the group of points in $A(\overline{\mathbf{Q}})$ which are annihilated by all elements of $\mathfrak{m}$. It is easy to check that $A[\mathfrak{m}]$ is free of rank two over $\mathbf{F}=R / \mathfrak{m}$, cf. [32, Prop. 10, p. 56]. In fact, the representation

$$
\rho: \operatorname{Gal}(\overline{\mathbf{Q}} / \mathbf{Q}) \rightarrow \mathbf{G L}(2, \mathbf{F})
$$

defined by the action of $\operatorname{Gal}(\overline{\mathbf{Q}} / \mathbf{Q})$ on $A[\mathfrak{m}]$ is just the mod $\mathfrak{m}$ reduction of the $\mathfrak{m}$-adic representation of $\operatorname{Gal}(\overline{\mathbf{Q}} / \mathbf{Q})$ which is attached to $A$. As usual, we denote by $p$ the characteristic of $\mathbf{F}$.

Proposition 5.1. Assume that $p$ is odd, that $\rho$ is irreducible, and that $A$ is semistable over $\mathbf{Q}$. Then $\rho$ is a semistable representation.

Proof. We first show that the determinant of $\rho$ is the mod $p$ cyclotomic character $\chi$. According to the statement of [23, Lemma 3.1], we have $\operatorname{det} \rho=$ $\epsilon \chi$, where $\epsilon$ is a Dirichlet character $\operatorname{Gal}(\overline{\mathbf{Q}} / \mathbf{Q}) \rightarrow R^{*}$ which depends only 
on $A$ (i.e., is independent of $\mathfrak{m}$ ) and which is ramified only at primes of bad reduction for $A$. In fact, the proof of this Lemma shows that $\epsilon$ is unramified at a prime $q$ whenever the following condition is satisfied: there is a prime $\lambda$ of $R$ such that the determinant of the $\lambda$-adic representation of $A$ is unramified at $q$. Now fix $q$ and take any prime $\lambda$ not dividing $q$. By a well known result of Grothendieck [9, Prop. 3.5], each element of an inertia subgroup for $q$ in $\operatorname{Gal}(\overline{\mathbf{Q}} / \mathbf{Q})$ acts unipotently in the $\lambda$-adic representation for $A$. In particular, the determinant of this representation is unramified at $q$. Thus $\epsilon$ is unramified at every prime number $q$, so that $\epsilon$ is the trivial character.

The same proposition of Grothendieck, applied to the $\mathfrak{m}$-adic representation for $A$, shows that the conductor of $\rho$ is square free. We may paraphrase this statement by saying that $\rho$ is semistable outside $p$. Furthermore, results of Raynaud [16] imply that $k(\rho)=2$, so that $\rho$ is semistable at $p$, whenever $\rho$ is finite at $p$. (See [28, Prop. 4, p. 189].) It remains only to show that $k(\rho)=p+1$ if $\rho$ is not finite at $p$.

For this, we consider $A[\mathfrak{m}]$ as a subgroup of $A[p]$, and view both as modules for a decomposition group $D_{p}$ in $\operatorname{Gal}(\overline{\mathbf{Q}} / \mathbf{Q})$ for the prime $p$. As explained in the proof of [21, Lemma 6.2], one may deduce from Grothendieck's study in SGA7I that $A[p]$ is an extension of an unramified $D_{p}$-module by a subgroup $A[p]^{\mathrm{f}}$ which is finite, i.e., which extends to a finite flat group scheme over $\mathbf{Z}_{p}$. Were $A[\mathfrak{m}]$ contained in $A[p]^{\mathrm{f}}, A[\mathfrak{m}]$ would be finite, contrary to assumption. Hence $A[\mathfrak{m}]$ has an unramified quotient. This implies that the restriction of $\rho$ to $D_{p}$ has the form $\left(\begin{array}{cc}\theta_{1} \chi & * \\ 0 & \theta_{2}\end{array}\right)$, where the $\theta_{i}$ are unramified characters. The recipe for $k(\rho)$ given in $[28, \S 2]$ then sets $k(\rho)=p+1$. (Compare Remarque (1) on page 188 of [28].)

\section{Application to $J_{0}(N)$ for prime $N$.}

Let $N$ be a prime number, and consider the abelian variety $J=J_{0}(N)$ over $\mathbf{Q}$. In this section and the next, we will study the action of $\operatorname{Gal}(\overline{\mathbf{Q}} / \mathbf{Q})$ on torsion points of $J$. The first work in this direction was the investigation of Shimura [30], which concerns the $\bmod p \operatorname{representation}$ of $\operatorname{Gal}(\overline{\mathbf{Q}} / \mathbf{Q})$ defined by $J_{0}(11)$, when $p$ lies between 7 and 97 . Shimura's discussion was completed by Serre $[26, \S 5.5]$, who determined for all $p$ the image of the $\bmod p$ representation attached to $J_{0}(11)$. Subsequently, Lang-Trotter [12], Part I, $\S 8$; calculated the image of the (adelic) representation of $\operatorname{Gal}(\overline{\mathbf{Q}} / \mathbf{Q})$ defined by all torsion points of $J_{0}(11)$.

In what follows, we generalize some of the results of Serre and LangTrotter to the case where 11 is replaced by an arbitrary prime. We exploit 
the insights of Mazur's article [13], which makes an extensive study of the action of $\operatorname{Gal}(\overline{\mathbf{Q}} / \mathbf{Q})$ on torsion points of $J_{0}(N)$, and the recent thesis of B. Kaskel [10], which determines the image of the adelic representation defined by $J_{0}(37)$. There is a certain amount of overlap with the joint article [2].

Let $\mathbf{T}$ be the ring of Hecke operators $\mathbf{Z}\left[\ldots, T_{n}, \ldots\right]$, considered as a ring of endomorphisms of $J_{0}(N)$. To orient the reader, we recall that $\mathbf{T}$ is the full ring of endomorphisms of $J_{0}(N)$ [13, p. 95]. To avoid the situation where $N$ is very small, we will assume that $J_{0}(N) \neq 0$. This means that $N=11$ or that $N \geq 17$.

Let $p$ be a prime number $\geq 5$.

Proposition 6.1. The quotient $\mathbf{T} / p \mathbf{T}$ is generated by the operators $T_{n}$ with $n$ prime to $p N$.

Proof. Let $S$ be the space of cusp forms of weight 2 on $\Gamma_{0}(N)$ over $\mathbf{F}_{p}$. We view $S$ as the $\bmod p$ reduction of the space of weight-two forms on $\Gamma_{0}(N)$ with integral $q$-expansions. (See [13, Ch. II, §4] for a comparison of several possible definitions of $S$.) We consider the bilinear pairing $\alpha: \mathbf{T} / p \mathbf{T} \times S \rightarrow$ $\mathbf{F}_{p}$ which maps $(T, f)$ to the initial coefficient of $q$ in the Fourier expansion of $f \mid T$. It is well known that $\alpha$ is a perfect pairing; this is explained, for example, in $[20, \S 1]$. To prove the Proposition, then, it suffices to prove that there is no non-zero element $f=\sum a_{n} q^{n}$ of $S$ which satisfies $a_{n}=0$ for all $n$ prime to $p N$.

Suppose that $f=\sum a_{n} q^{n}$ satisfies the condition. We will show first that $a_{n}=0$ for all $n$ prime to $N$. If $N=p$ there is nothing extra to prove, so we will assume for the moment that $N$ and $p$ are distinct. Let $g$ be the form $\sum_{(N, n)=1} a_{n} q^{n}$, i.e., the sum

$$
\sum_{n=1}^{\infty} b_{n} q^{n}, \quad b_{n}= \begin{cases}a_{n} & \text { if }(n, N)=1 \\ 0 & \text { otherwise }\end{cases}
$$

Then $b_{n}=0$ whenever $n$ is not a multiple of $p$; our aim is to show that $g=0$. Now the point is that $g$ may be considered as a weight-two mod $p$ modular form on $\Gamma_{0}\left(N^{2}\right)$; this follows from [31, Prop. 3.64]. The hypothesis about the vanishing of the $b_{n}$ means that $g$ is annihilated by the operator $\theta=q \frac{d}{d q}$. Since $p>2$, this forces $g=0$ as desired, since $\theta g$ has "filtration" $p+3$ if $g$ is non-zero. (This is Katz's generalization of the Serre-Swinnerton-Dyer theorem [11, p. 55].)

To complete the proof, we must show that $f=0$. This follows from Proposition 4.10, Lemma 5.9 and Lemma 5.10 of [13, Ch. II].

Remark. The argument we have given is essentially that of [13], Ch. II, Prop. 14.13. The exploitation of $q \frac{d}{d q}$ to deal with the absence of $T_{p}$ is a 
familiar ploy - it was used by the author in [22, Prop. 2] and by Wiles in [36, Lemma, p. 491].

Suppose now that $\mathfrak{m}$ is a maximal ideal of $\mathbf{T}$ of residue characteristic $p$. (We continue to assume $p \geq 5$.) Let $J[\mathfrak{m}]$ denote the kernel of $\mathfrak{m}$ on $J(\overline{\mathbf{Q}})$. By the results of $[13], J[\mathfrak{m}]$ defines the two-dimensional semisimple representation $\rho_{\mathfrak{m}}$ of $\operatorname{Gal}(\overline{\mathbf{Q}} / \mathbf{Q})$ which is associated with $\mathfrak{m}$; this representation is irreducible if and only if $\mathfrak{m}$ is not an Eisenstein prime of $\mathbf{T}$. When $\mathfrak{m}$ is non-Eisenstein, $\rho_{\mathfrak{m}}$ is a semistable representation: this follows from the results of Deligne-Rapoport [3] to the effect $J$ has multiplicative reduction at $N$, together with Proposition 5.1.

Proposition 6.2. The image of $\rho_{\mathfrak{m}}: \operatorname{Gal}(\overline{\mathbf{Q}} / \mathbf{Q}) \rightarrow \mathbf{G L}(2, \mathbf{T} / \mathfrak{m})$ is the group of elements of $\mathbf{G L}(2, \mathbf{T} / \mathfrak{m})$ having determinant in $\mathbf{F}_{p}^{*}$.

Proof. This mild strengthening of [13, Ch. II, Prop. 14.12] may be derived directly from Theorem 2.5. Indeed, for each prime $\ell$ different from $p$ and $N$, the image under $\rho_{\mathfrak{m}}$ of a Frobenius element for $\ell$ in $\operatorname{Gal}(\overline{\mathbf{Q}} / \mathbf{Q})$ has trace $T_{\ell} \bmod \mathfrak{m}$. It follows from the Lemma above that the $T_{\ell} \bmod \mathfrak{m}$ generate $\mathbf{T} / \mathfrak{m}$.

Remark. By using Theorem 2.6 in place of Theorem 2.5, we obtain the stronger statement that $\rho_{\mathfrak{m}}(X)$ is the group of matrices in $\mathbf{G L}(2, \mathbf{T} / \mathfrak{m})$ whose determinants lie in $\mathbf{F}_{p}^{*}$. A similar remark applies to the Proposition which follows.

Suppose next that $p \geq 5$ is such that none of the $\mathfrak{m} \mid p$ in $\mathbf{T}$ is an Eisenstein prime. This means simply that $N \not \equiv 1 \bmod p$. Let $\mathbf{F}$ be the $\mathbf{F}_{p}$-algebra $\prod_{m \mid p} \mathbf{T} / \mathfrak{m}$, and let

$$
\rho: \operatorname{Gal}(\overline{\mathbf{Q}} / \mathbf{Q}) \rightarrow \mathbf{G L}(2, \mathbf{F})
$$

be the product of the $\rho_{\mathfrak{m}}$.

Proposition 6.3. The image of $\rho$ is the group of matrices in $\mathbf{G L}(2, \mathbf{F})$ having determinant in $\mathbf{F}_{p}^{*}$.

Proof. Indeed, the natural map $\mathbf{T} / p \mathbf{T} \rightarrow \mathbf{F}$ is surjective by the Chinese remainder theorem. Accordingly, the Lemma implies that $\mathbf{F}$ is generated by the images of the $T_{\ell}$ with $\ell$ prime to $p N$. In the language of Theorem 3.2, this means that $\mathbf{F}^{\prime}=\mathbf{F}$. Applying that theorem, we find that the image of $\rho$ is as stated.

Continuing the discussion, we add the hypothesis that the surjection $\mathbf{T} / p \mathbf{T} \rightarrow \mathbf{F}$ is an isomorphism, i.e., that $p$ is unramified in $\mathbf{T}$. Then $\rho$ is the 
representation giving the action of $\operatorname{Gal}(\overline{\mathbf{Q}} / \mathbf{Q})$ on the group $J[p]$, viewed as a free $\mathbf{T} / p \mathbf{T}$-module of rank two. Proposition 6.3 thus furnishes a description of the Galois group of the field cut out by the $p$-division points of $J$. Further, as we have seen in Proposition 4.2, the result of Proposition 6.3 is equivalent to an analogous statement about the $p$-adic Galois representation defined by $J$. More precisely, because $p$ in unramified in $\mathbf{T}, \mathbf{T} \otimes \mathbf{Z}_{p}$ is a product of discrete valuation rings. The Tate module $\operatorname{Ta}_{p}(J)$ of $J$ is then automatically free of rank two over $\mathbf{T} \otimes \mathbf{Z}_{p}$. After choosing a basis of $\operatorname{Ta}_{p}(J)$, we may summarize the action of $\operatorname{Gal}(\overline{\mathbf{Q}} / \mathbf{Q})$ on the $p$-power division points of $J$ by a homomorphism

$$
\tilde{\rho}: \operatorname{Gal}(\overline{\mathbf{Q}} / \mathbf{Q}) \rightarrow \mathbf{G L}\left(2, \mathbf{T} \otimes \mathbf{Z}_{p}\right) .
$$

By Propositions 4.2 and 6.3, we find that the image of $\tilde{\rho}$ is "as large as possible":

Theorem 6.4. Suppose that $p$ is unramified in $\mathbf{T}$ and that $p$ is prime to $6 \cdot(N-1)$. Then $\tilde{\rho}(\operatorname{Gal}(\overline{\mathbf{Q}} / \mathbf{Q}))=\left\{M \in \mathbf{G L}\left(2, \mathbf{T} \otimes \mathbf{Z}_{p}\right) \mid \operatorname{det} M \in \mathbf{Z}_{p}^{*}\right\}$.

Remark. We obtain the more precise equality

$$
\tilde{\rho}(X)=\left\{M \in \mathbf{G L}\left(2, \mathbf{T} \otimes \mathbf{Z}_{p}\right) \mid \operatorname{det} M \in \mathbf{Z}_{p}^{*}\right\}
$$

from Theorem 3.4 and Proposition 4.2.

\section{Complements.}

We continue our study of $J=J_{0}(N)$, where $N$ is a prime number by studying products of $p$-adic representations attached to $J$. We are motivated by the discussions of $[25, \mathrm{Ch} . \mathrm{IV}, \S 3]$ and $[26, \S 4.4]$, whose tools serve very well in this context.

For each prime $p$, let $\mathrm{Ta}_{p}(J)$ be the $\mathbf{Z}_{p}$-adic Tate module of $J$ and write $\rho_{p}$ (rather than $\tilde{\rho}$, as above) for the $p$-adic representation of $\mathrm{Gal}(\overline{\mathbf{Q}} / \mathbf{Q})$ which is associated to $J$ :

$$
\tilde{\rho}: \operatorname{Gal}(\overline{\mathbf{Q}} / \mathbf{Q}) \rightarrow \operatorname{Aut}_{\mathbf{T} \otimes \mathbf{z}_{p}}\left(\operatorname{Ta}_{p}(J)\right) \subset \operatorname{Aut}_{\mathbf{T} \otimes \mathbf{Q}_{p}}\left(\operatorname{Ta}_{p}(J) \otimes \mathbf{Q}\right) .
$$

Let $G_{p}$ be the image of $\rho_{p}$. This group was determined in Theorem 6.4 for most prime numbers $p$. We shall describe the $p$-adic Lie algebra of $G_{p}$ in general; thus we determine $G_{p}$ "up to finite groups" even when (or especially when) $p$ does not satisfy the hypothesis to Theorem 6.4.

We recall that Mazur proved [13, Ch. II, $\S 15-\S 17]$ that $\mathrm{Ta}_{p}(J)$ is free of rank two over $\mathbf{T} \otimes \mathbf{Z}_{p}$ when $p$ is odd, and also in many circumstances when $p=2$. In this favorable situation, we may view $G_{p}$ as a closed subgroup 
of $\mathbf{G L}\left(2, \mathbf{T} \otimes \mathbf{Z}_{p}\right)$. In any case, $\operatorname{Ta}_{p}(J) \otimes \mathbf{Q}_{p}$ is free of rank two over the $\mathbf{Q}_{p^{-}}$ algebra $\mathbf{T} \otimes \mathbf{Q}_{p}$ by [13, Ch. II, Lemma 7.7]. Hence we may always regard $G_{p}$ as a closed subgroup of $\mathbf{G L}(2, E)$, where $E=\mathbf{T} \otimes \mathbf{Q}_{p}$. Note that $E$ is a commutative semisimple $\mathbf{Q}_{p}$-algebra, i.e., the product of fields which are finite extensions of $\mathbf{Q}_{p}$. Because the determinant of $\rho_{p}$ is the $p$-adic cyclotomic character, we have $G_{p} \subseteq H_{p}$, where

$$
H_{p}:=\left\{M \in \mathbf{G L}(2, E) \mid \operatorname{det} M \in \mathbf{Q}_{p}^{*}\right\} .
$$

Proposition 7.1. The group $G_{p}$ is open in $H_{p}$.

Proof. Let $\mathfrak{g}$ and $\mathfrak{h}$ be the $p$-adic Lie algebras of $G_{p}$ and $H_{p}$, respectively. Thus

$$
\mathfrak{g} \subseteq \mathfrak{h}=\mathbf{Q}_{p} \times \mathfrak{s l}_{2}(E),
$$

where $\mathfrak{s l}_{2}(E)=[\mathfrak{h}, \mathfrak{h}]$ is the Lie algebra of two-by-two matrices over $E$ with trace 0 . Since the $p$-adic cyclotomic character has infinite order, $g$ is not contained in $\mathfrak{s l}_{2}(E)$. The proposition states that $\mathfrak{g}=\mathfrak{h}$.

The equality $\mathfrak{g}=\mathfrak{h}$ is proved as $[18$, Th. 4.5.4] in the special case where $\mathbf{T} \otimes \mathbf{Q}$ is a field, i.e., where $J$ is a simple abelian variety. (Note that $\operatorname{End}_{\overline{\mathbf{Q}}} J=$ $\operatorname{End}_{\mathbf{Q}} J$, as was proved in [17]; hence $J$ is simple over $\mathbf{Q}$ if and only if it is absolutely simple.) In the general case, $J$ is isogenous over $\mathbf{Q}$ to a product $A_{1} \times \cdots \times A_{t}$ of simple abelian varieties to which [18, Th. 4.5.4] applies. Thus $\mathfrak{g}$ and $\mathfrak{h}$ have equal images in $\operatorname{End}\left(\operatorname{Ta}_{p}\left(A_{i}\right) \otimes \mathbf{Q}_{p}\right)$ for each $i$. Moreover, one knows that $\operatorname{End}_{\mathfrak{g}}\left(\operatorname{Ta}_{p}(J)\right)=E=\operatorname{End}_{\mathfrak{h}}\left(\operatorname{Ta}_{p}(J)\right)$. Indeed, the Tate conjecture for abelian varieties, which was proved in [5], implies that $\operatorname{End}_{\mathfrak{g}}\left(\operatorname{Ta}_{p}(J)\right)=\left(\operatorname{End}_{\overline{\mathbf{Q}}} J\right) \otimes \mathbf{Q}_{p}$. On the other hand, one knows by [17] that $\left(\operatorname{End}_{\overline{\mathbf{Q}}} J\right) \otimes \mathbf{Q}=\mathbf{T} \otimes \mathbf{Q}$; hence $\left(\operatorname{End}_{\overline{\mathbf{Q}}} J\right) \otimes \mathbf{Q}_{p}=E$, as was claimed. The proof of $[18$, Th. 4.4.10] now yields the required equality $\mathfrak{g}=\mathfrak{h}$.

For each set of prime numbers $S$, let

$$
\rho_{S}: \operatorname{Gal}(\overline{\mathbf{Q}} / \mathbf{Q}) \rightarrow \prod_{p \in S} \operatorname{Aut}\left(\operatorname{Ta}_{p}(J)\right)
$$

be the product of the $\rho_{p}$ for $p$ in $S$. Let $G_{S}$ be the image of $\rho_{S}$, so that $G_{S}$ is a closed subgroup of the product $\prod_{p \in S} G_{p}$.

Corollary 7.2. If $S$ is a finite set of primes, then $G_{S}$ is open in $H_{S}:=$ $\prod_{p \in S} H_{p}$.

Proof. The assertion to be proved follows from Proposition 7.1 and an argument due to Serre [25, Lemma 4, p. IV-24]:

Fix $p \in S$ for the moment, and ensure by a change of basis if necessary that $G_{p}$ is a subgroup of $\mathbf{G} \mathbf{L}(2, R)$, where $R$ is the ring of integers of $E$. Let 
$\mathbf{F}$ be the product of the residue fields of the factors of $R$, and let $G_{p}^{\prime}$ be the kernel of the composition

$$
G_{p} \hookrightarrow \mathbf{G L}(2, R) \rightarrow \mathbf{G L}(2, F) .
$$

Clearly, $G_{p}^{\prime}$ has finite index in $G_{p}$, so that $G_{p}^{\prime}$ is open in $H_{p}$ by Proposition 7.1. Further, $G_{p}^{\prime}$ is a pro- $p$ group; in fact, it is a projective limit of nilpotent groups of $p$-power order.

Let $G_{S}^{\prime}$ be the inverse image of $\prod_{p \in S} G_{p}^{\prime}$ in $G_{S}$. Since $G_{S}^{\prime}$ is a subgroup of $\prod_{p \in S} G_{p}^{\prime}$, it is pro-nilpotent. Thus $G_{S}^{\prime}$ is the product of its Sylow subgroups. Now the $p$-Sylow subgroup of $G_{S}^{\prime}$ has finite index in $G_{p}$. Thus $G_{S}^{\prime}$ has finite index in $\prod_{p \in S} G_{p}$, a group which is open in $H_{S}$ by Proposition 7.1. Hence $G_{S}$ is open in $H_{S}$.

Theorem 7.3 (Kaskel [10]). Assume that $N$ does not belong to $S$. Then $G_{S}=\prod_{p \in S} G_{p}$.

Proof. We first consider the case where $S$ is finite, arguing by induction on the size of $S$. The statement to be proved is evident if $S$ has at most one element, so we may assume that $S=T \coprod\{p\}$ and that the statement is true with $S$ replaced by $T$. We must show that the natural injection $G_{S} \hookrightarrow G_{T} \times G_{p}$ is an isomorphism, or equivalently that the Galois extensions of $\mathbf{Q}$ cut out by $\rho_{T}$ and $\rho_{p}$ are linearly disjoint. Let $K$ be the intersection of these two fields. Then $K$ is ramified only at $N$, since $\rho_{p}$ is ramified only at $N$ and at $p$, while $\rho_{T}$ is unramified at $p$. Further, the inertia groups for the prime $N$ in the image of $\rho_{p}$ are pro- $p$ groups since $J_{0}(N)$ is semistable at $N$. Similarly, the inertia groups for $N$ in the image of $\rho_{T}$ are profinite groups of order prime to $p$; indeed, $p$ is not a member of $T$. Hence the inertia groups for $N$ in $\operatorname{Gal}(K / \mathbf{Q})$ are trivial, so that $K$ is an everywhere unramified extension of $\mathbf{Q}$. This gives $K=\mathbf{Q}$ and proves the linear disjointness.

The case where $S$ is not necessarily finite now follows, since $G_{S}$ is closed and dense in $\prod_{p \in S} G_{p}$.

Corollary 7.4. Let $S$ be the set of prime numbers $p$ which are prime both to $6(N-1)$ and to the discriminant of $\mathbf{T}$. Then

$$
G_{S}=\prod_{p \in S}\left\{M \in \mathbf{G L}\left(2, \mathbf{T} \otimes \mathbf{Z}_{p}\right) \mid \operatorname{det} M \in \mathbf{Z}_{p}^{*}\right\} .
$$

Proof. We shall give two proofs of this result, the first in spirit of Kaskel's theorem. For each $p \in S$, we have $G_{p}=\left\{M \in \mathbf{G L}\left(2, \mathbf{T} \otimes \mathbf{Z}_{p}\right) \mid \operatorname{det} M \in \mathbf{Z}_{p}^{*}\right\}$ by Theorem 6.4. Let $T$ be the set of primes $p \in S$ which are different from $N$; thus $S=T \amalg\{N\}$ if $N$ is prime to the discriminant of $\mathbf{T}$ and $S=T$ if not. 
We clearly have $G_{T}=\prod_{p \in T}\left\{M \in \mathbf{G L}\left(2, \mathbf{T} \otimes \mathbf{Z}_{p}\right) \mid \operatorname{det} M \in \mathbf{Z}_{p}^{*}\right\}$ by Kaskel's theorem and Theorem 6.4. This gives what is wanted if $S=T$. In what follows, we suppose to the contrary that $N$ belongs to $S$.

The idea now is to analyze the subgroup $G_{S}$ of $G_{T} \times G_{N}$ as in the proof above. Let $K / \mathbf{Q}$ now be the obstruction to the equality $G_{S}=G_{T} \times G_{N}$. In other words, $K$ is the intersection of the two extensions of $\mathbf{Q}$ whose Galois groups are the images of $G_{T}$ and $G_{N}$. Clearly, $K$ is ramified only at $N$. Moreover, $I$ is an inertia group for $N$ in $\operatorname{Gal}(K / \mathbf{Q})$, then the order of $I$ (as a supernatural number) is divisible only by the primes in $T$. In particular, this order is prime to $N(N-1)$. On the other hand, it is easy to see that only $N$ and primes dividing $N-1$ can intervene in the order of an inertia group for $N$ in the image of $\rho_{N}$. Indeed, the restriction of $\rho_{N}$ to an inertia group

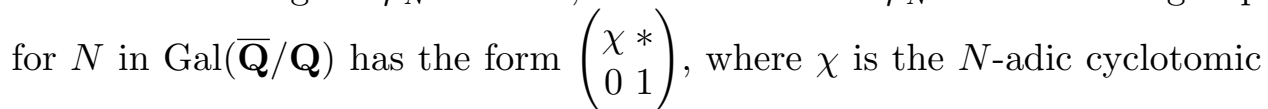
character. Hence $K$ is unramified at $N$, and we obtain the required equality $G_{S}=G_{T} \times G_{N}$ as in the proof of Kaskel's theorem.

To make a second proof of the equality

$$
G_{S}=\prod_{p \in S}\left\{M \in \mathbf{G L}\left(2, \mathbf{T} \otimes \mathbf{Z}_{p}\right) \mid \operatorname{det} M \in \mathbf{Z}_{p}^{*}\right\},
$$

we fix a prime $p$ in $T$ and let $X$ be the group which we considered in Theorem 2.6 and Theorem 3.4. We have $\rho_{p}(X)=\left\{M \in \mathbf{G L}\left(2, \mathbf{T} \otimes \mathbf{Z}_{p}\right) \mid \operatorname{det} M \in\right.$ $\left.\mathbf{Z}_{p}^{*}\right\}$ as explained in the remark at the end of the preceding section. On the other hand, if $p^{\prime}$ is an element of $S$ which is different from $p, \rho_{p^{\prime}}$ is unramified at $p$ and therefore $\rho_{p^{\prime}}(X)=\{1\}$. Thus $\rho_{S}(X)$ contains

$$
\left\{M \in \mathbf{G L}\left(2, \mathbf{T} \otimes \mathbf{Z}_{p}\right) \mid \operatorname{det} M \in \mathbf{Z}_{p}^{*}\right\},
$$

viewed as a subgroup of the product. Hence we have

$$
G_{S} \supseteq \prod_{p \in T}\left\{M \in \mathbf{G L}\left(2, \mathbf{T} \otimes \mathbf{Z}_{p}\right) \mid \operatorname{det} M \in \mathbf{Z}_{p}^{*}\right\} .
$$

This gives what is wanted if $S=T$, so we assume once again that $N$ belongs to $S$. Then $G_{S}$ is a subgroup of the product

$$
\prod_{p \in S}\left\{M \in \mathbf{G L}\left(2, \mathbf{T} \otimes \mathbf{Z}_{p}\right) \mid \operatorname{det} M \in \mathbf{Z}_{p}^{*}\right\}
$$

which maps onto $\left\{M \in \mathbf{G L}\left(2, \mathbf{T} \otimes \mathbf{Z}_{N}\right) \mid \operatorname{det} M \in \mathbf{Z}_{N}^{*}\right\}$ by Theorem 6.4. Moreover $G_{S}$ contains the kernel of the natural projection

$$
\begin{aligned}
\prod_{p \in S}\left\{M \in \mathbf{G L}\left(2, \mathbf{T} \otimes \mathbf{Z}_{p}\right) \mid \operatorname{det} M\right. & \left.\in \mathbf{Z}_{p}^{*}\right\} \\
& \rightarrow\left\{M \in \mathbf{G L}\left(2, \mathbf{T} \otimes \mathbf{Z}_{N}\right) \mid \operatorname{det} M \in \mathbf{Z}_{N}^{*}\right\} .
\end{aligned}
$$


Therefore $G_{S}$ is the full product.

For our final result, we consider the product $\rho_{\mathrm{f}}$ of all of the representations $\rho_{p}$, i.e., the representation $\rho_{S}$ where $S$ is the set of all prime numbers. We view $\rho_{\mathrm{f}}$ as taking values in

$$
\left\{M \in \mathbf{G L}\left(2, \mathbf{T} \otimes \mathbf{Q}_{2}\right) \mid \operatorname{det} M \in \mathbf{Q}_{2}^{*}\right\} \times \prod_{p \neq 2}\left\{M \in \mathbf{G L}\left(2, \mathbf{T} \otimes \mathbf{Z}_{p}\right) \mid \operatorname{det} M \in \mathbf{Z}_{p}^{*}\right\},
$$

a group that we will call $\mathcal{A}$. (We separate 2 from the odd primes since it is not known that $\mathrm{Ta}_{2}$ is free of rank two over $\mathbf{T} \otimes \mathbf{Z}_{2}$.)

Theorem 7.5. The image of $\rho_{\mathrm{f}}$ is an open subgroup of $\mathcal{A}$.

Proof. Let $\mathcal{A}_{p}$ be the $p$ th component of $\mathcal{A}$, so that we have $\mathcal{A}=\prod \mathcal{A}_{p}$, with the product extended over all primes. Let $S$ be the set of those $p$ which are prime to $6(N-1) N$ and to the discriminant of $\mathbf{T}$. Fix $p \in S$ and let $X$ be the subgroup of $\operatorname{Gal}(\overline{\mathbf{Q}} / \mathbf{Q})$ which we have considered repeatedly: the closed subgroup of $\operatorname{Gal}(\overline{\mathbf{Q}} / \mathbf{Q})$ generated by all inertia groups for $p$. As we have seen, $\rho_{p}(X)=\mathcal{A}_{p}$; on the other hand $\rho_{p^{\prime}}(X)=\{1\}$ for $p^{\prime} \neq p$. Hence $\rho_{\mathrm{f}}(X)=\mathcal{A}_{p}$, where $\mathcal{A}_{p}$ is considered as a subgroup of the product $\mathcal{A}$. On varying $p$, we find that $\rho_{\mathrm{f}}(\operatorname{Gal}(\overline{\mathbf{Q}} / \mathbf{Q}))$ contains the group $\prod_{p \in S} \mathcal{A}_{p}$, i.e., the kernel of the projection $\mathcal{A} \rightarrow \prod_{p \notin S} \mathcal{A}$. On the other hand, the image of $\mathrm{Gal}(\overline{\mathbf{Q}} / \mathbf{Q})$ in this finite product is open by Corollary 7.2. Hence $\rho_{\mathrm{f}}(\operatorname{Gal}(\overline{\mathbf{Q}} / \mathbf{Q}))$ is open in the full product.

\section{References}

[1] C. Batut, D. Bernardi, H. Cohen and M. Olivier, GP/PARI, Available by anonymous ftp from megrez.math.u-bordeaux.fr or math.ucla.edu, in the directory /pub/pari.

[2] R. Coleman, B. Kaskel and K. Ribet, Torsion points on $X_{0}(N)$, Contemporary Math., to appear.

[3] P. Deligne and M. Rapoport, Les schémas de modules de courbes elliptiques, Lecture Notes in Math., Vol. 349, Springer-Verlag, Berlin and New York, (1973), 143-316.

[4] L.E. Dickson, Linear groups with an exposition of the Galois field theory, Dover, New York, 1958.

[5] G. Faltings, Endlichkeitssätze für abelsche Varietäten über Zahlkörpern, Invent. Math., 73 (1983), 349-366.

[6] G. Frey (Ed.) On Artin's conjecture for odd 2-dimensional representations, Lecture Notes in Math., Vol. 1585, Springer-Verlag, Berlin-Heidelberg-New York, 1994.

[7] D. Gorenstein, Finite Groups, Chelsea, New York, 1980. 
[8] B.H. Gross, A tameness criterion for Galois representations associated to modular forms $\bmod p$, Duke Math. J., 61 (1990), 445-517.

[9] A. Grothendieck, SGA7 I, Exposé IX, Lecture Notes in Math., Vol. 288, SpringerVerlag, Berlin and New York, (1972), 313-523.

[10] B. Kaskel, The adelic representation associated to $X_{0}(37), \mathrm{PhD}$. thesis, UC Berkeley, May, 1996.

[11] N.M. Katz, A result on modular forms in characteristic $p$, Lecture Notes in Math., Vol. 601, Springer-Verlag, Berlin and New York, (1977), 53-61.

[12] S. Lang and H. Trotter, Frobenius distributions in $\mathbf{G L}_{2}$-extensions, Lecture Notes in Math., Vol. 504, Springer-Verlag, Berlin and New York, 1976.

[13] B. Mazur, Modular curves and the Eisenstein ideal, Publ. Math. IHES, 47 (1977), 33-186.

[14] T. Miyake, Modular forms, Springer-Verlag, Berlin and New York, 1989.

[15] J. Oesterlé, Travaux de Wiles (et Taylor, ... ), Partie II, Séminaire Bourbaki, 804 (1995).

[16] M. Raynaud, Schémas en groupes de type $(p, \ldots, p)$, Bull. Soc. Math. France, 102 (1974), 241-280.

[17] K.A. Ribet, Endomorphisms of semi-stable abelian varieties over number fields, Annals of Math., 101 (1975), 555-562.

[18] Galois action on division points of abelian varieties with many real multiplications, Am. J. Math., 98 (1976), 751-804.

[19] - On $\ell$-adic representations attached to modular forms, Invent. Math., 28 (1975), 245-275.

[20] Mod $p$ Hecke operators and congruences between modular forms, Invent. Math., 71 (1983), 193-205.

[21] - On modular representations of $\mathrm{Gal}(\overline{\mathbf{Q}} / \mathbf{Q})$ arising from modular forms, Invent. Math., 100 (1990), 431-476.

$[22]$, Multiplicities of $p$-finite mod $p$ Galois representations in $J_{o}(N p)$, Boletim da Sociedade Brasileira de Matemática, Nova Série, 21 (1991), 177-188.

[23] - Abelian varieties over $\mathbf{Q}$ and modular forms, 1992 Proceedings of KAIST Mathematics Workshop, Taejon, Korea Advanced Institute of Science and Technology, (1992), 53-79.

[24] I. Schur, Arithmetische Untersuchungen über endliche Gruppen linearer Substitutionen, Sitz. Pr. Akad. Wiss., (1906), 164-184; Gesam. Abhl., I, 177-197, SpringerVerlag, Berlin-Heidelberg-New York-Tokyo, 1973.

[25] J.-P. Serre, Abelian $\ell-a d i c$ representations and elliptic curves, Addison-Wesley Publ. Co., Redding, Mass., 1989.

[26] - Propriétés galoisiennes des points d'ordre fini des courbes elliptiques, Invent. Math., 15 (1972), 259-331.

[27] _ Euvres, Springer-Verlag, Berlin-Heidelberg-New York-Tokyo, 1986.

[28] Sur les représentations modulaires de degré 2 de $\operatorname{Gal}(\overline{\mathbf{Q}} / \mathbf{Q})$, Duke Math. J., 54 (1987), 179-230.

[29] Travaux de Wiles (et Taylor, ... ) , Partie I, Séminaire Bourbaki, 803 (1995). 
[30] G. Shimura, A reciprocity law in non-solvable extensions, Journal für die reine und angewandte Mathematik, 221 (1966), 209-220.

[31] , Introduction to the arithmetic theory of automorphic functions, Princeton University Press, Princeton, 1971.

[32] G. Shimura and Y. Taniyama, Complex multiplication of abelian varieties and its applications to number theory, Second printing corrected, Math. Society of Japan, Tokyo, 1975.

[33] J. Sturm, On the congruence of modular forms, Lecture Notes in Math., Vol. 1240, Springer-Verlag, Berlin and New York, (1987), 275-280.

[34] J. Tate, The non-existence of certain Galois extensions of $\mathbf{Q}$ unramified outside 2, Contemporary Mathematics, 174 (1994), 153-156.

[35] J.-L. Waldspurger, Quelques propriétés arithmétiques de certaines formes automorphes sur GL(2), Compositio Math., 54 (1985), 121-171.

[36] A. Wiles, Modular elliptic curves and Fermat's Last Theorem, Annals of Math., 141 (1995), 443-551.

This article was begun while the author was a research professor at the MSRI, where research was supported in part by NSF grant DMS-9022140. This work was further supported by the investigator's NSF Grants DMS 88-06815 and DMS 93-06898. The author thanks the referee for a number of interesting suggestions and questions and Ian Kiming and Jean-François Mestre for carrying out computations in connection with the example discussed at the end of $\S 2$.

UNIVERSITY OF CALIFORNIA

Berkeley, CA 94720-3840 USA

E-mail address: ribet@math.berkeley.edu 